\title{
AN INVERSE PROBLEM FOR CIRCLE PACKING AND CONFORMAL MAPPING
}

\author{
ITHIEL CARTER AND BURT RODIN
}

\begin{abstract}
Figure 1.1 illustrates the fact that if a region is almost packed with circles of radius $\varepsilon$ in the hexagonal pattern and if the unit disk is packed in an isomorphic pattern with circles of varying radii then, after suitable normalization, the correspondence of circles converges to the Riemann mapping function as $\varepsilon \rightarrow 0$ (see [15]). In the present paper an inverse of this result is obtained as illustrated by Figure 1.2; namely, if the unit disk is almost packed with $\varepsilon$ circles there is an isomorphic circle packing almost filling the region such that, after suitable normalization, the circle correspondence converges to the conformal map of the disk onto the region as $\varepsilon \rightarrow 0$. Note that this set up yields an approximate triangulation of the region by joining the centers of triples of mutually tangent circles. Since this triangulation is intimately related to the Riemann mapping it may be useful for grid generation [18].
\end{abstract}

\section{INTRODUCTION}

In [15] it is shown that the Riemann mapping function $f$ from a bounded simply-connected region $R$ to the unit disk $\mathbb{D}$ can be approximated by circle packings. That is, if $R$ is filled with circles of radius $\varepsilon$ packed together in the hexagonal pattern then there is an isomorphic circle packing of $\mathbb{D}$ (see Figure 1.1) and, after suitable normalization, this isomorphism converges to the Riemann mapping function $f: R \rightarrow \mathbb{D}$ as $\varepsilon \rightarrow 0$.

One would like a similar result for the inverse function of $f$. That is, one wants to obtain a picture such as that in Figure 1.2 in which $\varepsilon$ circles are packed in $\mathbb{D}$, an isomorphic circle packing of a region $R$ is created, and this isomorphism converges to $f^{-1}: \mathbb{D} \rightarrow R$ as $\varepsilon \rightarrow 0$. Note that this set up yields an approximate triangulation of $R$; namely, the triangles of centers of triples of mutually tangent circles in $R$. This triangulation is intimately related to the Riemann mapping and therefore might be useful for grid generation (cf. [18]).

In Theorem 1, which first appeared in Carter [7], we construct circle packings having prescribed patterns and having prescribed radii for the border circles; the carrier can be a bordered surface of genus 0 or 1 . For genus 0 the statement

Received by the editors September 25, 1990.

1980 Mathematics Subject Classification (1985 Revision). Primary 30A30, 52A45.

Key words and phrases. Discrete conformal geometry, circle packing, numerical conformal mapping, grid generation.

This work was done during 1987-1988 and was supported by the NSF and DARPA; the results appear in the first-named author's 1989 doctoral dissertation at the University of California, San Diego. 

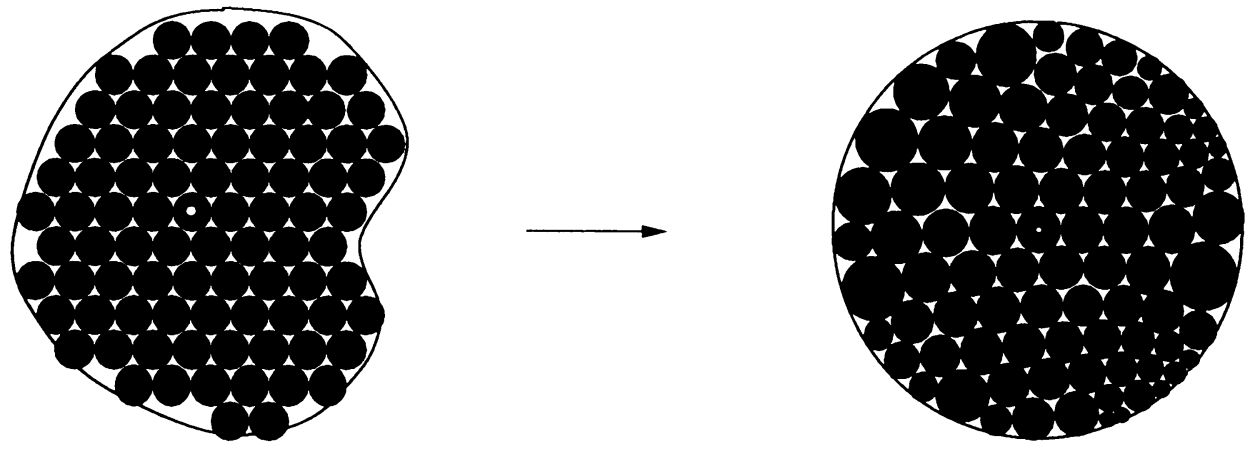

FIGURE 1.1. An approximate Riemann mapping
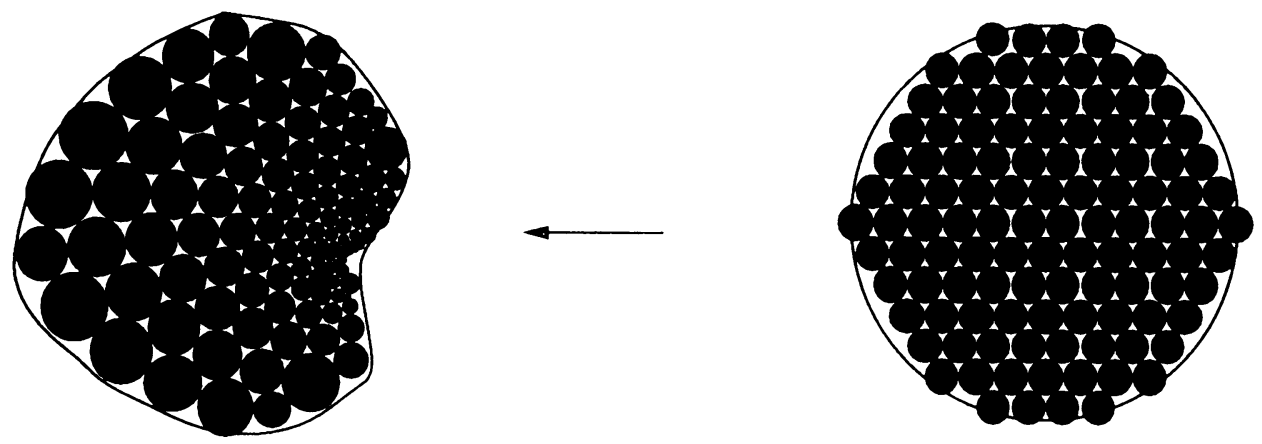

FIGURE 1.2. An approximate inverse Riemann mapping

of this result generalizes the theorem of Andreev [2] and Thurston [20] on the existence of circle packings on the sphere; the proof, however, makes essential use of that theorem. We were unable to give a proof using Thurston's continuity method as in [19 and 13]. Our proof adapts Thurston's algorithm to this more general case; we then use Peter Doyle's unpublished ideas [8] for proving the convergence of Thurston's algorithm to prove the theorem. The proof of Theorem 1 does indeed show that Thurston's algorithm converges in the genus zero case, but see Remarks 2 and 3 concerning its failure to converge in other cases.

Consider the following situation. Let $\Omega$ be a bounded, simply-connected domain and let $\rho$ be a positive continuous function on $\partial \Omega$. There is a unique suitably normalized conformal mapping $F$ of $\Omega$ with the property that $\left|F^{\prime}\right|$ extends continuously to the boundary and agrees with $\rho$ there. We wish to approximate $F$ by means of circle packing isomorphisms. To that end, consider the regular hexagonal circle packing $H_{\varepsilon}$ of the plane by circles of radius $\varepsilon$. Roughly speaking, let $C_{\varepsilon}$ consists of those circles from $H_{\varepsilon}$ which lie in $\Omega$. To each circle on the border of $C_{\varepsilon}$ assign the value of $\rho$ at a nearest point on $\partial \Omega$. Theorem 1 shows that there is an essentially unique circle packing immersion $C_{\varepsilon}^{\prime}$ which is isomorphic to $C_{\varepsilon}$ and which has the property that the ratio of the radii of each boundary circle in $C_{\varepsilon}^{\prime}$ to that of its corresponding circle in $C_{\varepsilon}$ is the assigned value $\rho$. Theorem 4 shows that this circle correspondence approximates $F$ in the sense that the isomorphism $C_{\varepsilon} \rightarrow C_{\varepsilon}^{\prime}$ converges to $F$ as $\varepsilon \rightarrow 0$.

The proof of Theorem 4 uses discrete potential theory and He's Theorem 
[10] that the hexagonal circle packing constants $s_{n}$ are of order $O(1 / n)$. An error term in the approximation of $F$ by circle packings is estimated (Theorem 3 ) in terms of some constants $\sigma_{n}$ which are analogous to $s_{n}$ but apply to circle packing immersions rather than imbeddings (see (2.1), (2.2), and Remark 1). In theorem 2 we prove that $\sigma_{n} \rightarrow 0$. The methods of Aharonov [1] can be used to obtain the estimate $\sigma_{n}=O(1 / n)$ under certain conditions.

Assume one is given a bounded, simply-connected region $R$. In order to approximate the conformal mapping $f^{-1}: \mathbb{D} \rightarrow R$ by the methods of this paper (Figure 1.2) it is necessary to estimate the function $\rho=\left|d f^{-1} / d z\right|$ on the boundary of the disk $\mathbb{D}$. One way of doing this is to first approximate the conformal mapping $R \rightarrow \mathbb{D}$ by the circle packing method of Figure 1.1 and use that information to approximate $\rho$. An integral equation for determining $\rho$ exactly is given in Warschawski [ 21 ; the equation for $\theta^{\prime} \equiv \rho$ appears in the proof of Theorem 4]; see Henrici [11, Theorem 16.7a, p. 395] for further discussion of Warschawski's equation.

\section{Definitions AND STATEMENT OF RESUlts}

For later reference we record the following fact.

Proposition 1 (The normalized conformal immersion determined by $\rho$ ). Let $\Omega$ be a bounded, simply-connected domain containing fixed points $z_{0}$ and $z_{1}$ and let $\rho$ be a positive continuous function on $\partial \Omega$. Then there is a unique conformal immersion $F: \Omega \rightarrow \mathbb{C}$, normalized by $F\left(z_{0}\right)=0$ and $F\left(z_{1}\right)>0$, such that $\left|F^{\prime}\right|$ extends continuously to the boundary of $\Omega$ and agrees with $\rho$ there.

If $u$ is the solution to the Dirichlet problem on $\Omega$ for the boundary values $\log \rho$ then $F$ can be obtained as $F=e^{i \theta} \int e^{u+i u^{*}} d z+c$, where $u^{*}$ is the conjugate harmonic function of $u$. The term conformal immersion is used to mean that the mapping $F$ has nonvanishing derivative but need not be one-toone. Conditions on $\rho$ which force $F$ to be univalent were considered in John [12] for the case of the unit disk as domain.

Let $T$ be a triangulation whose carrier is a compact surface with border. A radius function $R$ on $T$ is a positive function defined on the vertices of $T$. At each interior vertex $v_{i} \in T$ we define the curvature of $R$ at $v_{i}$ as follows. Associate to each face of the triangulation $T$, with vertices $\left(v_{i}, v_{j}, v_{k}\right)$ say, the Euclidean triangle determined by the centers of three mutually tangent circles which have radii $R\left(v_{i}\right), R\left(v_{j}\right), R\left(v_{k}\right)$. These triangles can be welded along corresponding edges to form a cone manifold; see Thurston [19]. Consider all faces which have $v_{i}$ as one of their vertices. Let $\theta_{1}, \theta_{2}, \ldots, \theta_{n}$ be the angles at $v_{i}$ in each of the corresponding Euclidean triangles. Then the curvature of $R$ at $v_{i}$ is $\kappa_{R}\left(v_{i}\right)=2 \pi-\left(\theta_{1}+\theta_{2}+\cdots+\theta_{n}\right)$. If $\kappa_{R}(v)=0$ for all interior vertices $v \in$ $T$ then we say that the radius function $R$ is flat. (A radius function $R$ may also be used to construct hyperbolic rather than Euclidean triangles. To emphasize the distinction one could speak of Euclidean, as opposed to hyperbolic, radius functions. Thurston [19] showed that a triangulated closed surface of genus greater than 1 supports a flat hyperbolic radius function. Beardon-Stevenson [5, Theorem 3] modified our Theorem 1, in the simply-connected case, from Euclidean to hyperbolic radius functions which can take the value $+\infty$. In the present paper the term radius function is always understood to mean Euclidean radius function.) 
If the carrier of $T$ is simply connected then a flat radius function $R$ determines a circle packing immersion uniquely up to a rigid motion (see [14]). Namely, for each vertex $v$ of $T$ place a circle of radius $R(v)$ in the plane $\mathbb{C}$ in such a way that if two vertices are joined by an edge then the corresponding circles in $\mathbb{C}$ are tangent. If this placement is done sequentially the position of the first circle is arbitrary, the position of the second is determined up to a rotation about the center of the first, and the positions of the remaining circles are completely determined. Let $P$ be the map which assigns to the vertex $v$ in $T$ the circle in $\mathbb{C}$ of radius $R(v)$ which corresponds to $v$ under this placement. We shall refer to $P$ as a circle packing immersion determined by $R$. We call $P$ a circle packing imbedding if the interiors of all of the disks in $\mathbb{C}$ are disjoint. The triangulation $T$ is called the nerve of $P$. Two packings are said to be isomorphic or combinatorially equivalent if their nerves are isomorphic. If the boundary of $T$ has three edges, and if $T$ is simply connected as in the present discussion, then the image circles of $P$ can be considered as a classical circle packing of $\mathbb{C} \cup\{\infty\}$; that is, $P$ is a circle packing imbedding whose nerve is a triangulation of the 2-sphere.

For $h>0$, we denote the hexagonal lattice of mesh $h$ by $\operatorname{HL}(h, \infty)$; thus $\mathrm{HL}(h, \infty)=\{(a+b \omega) h: a \in \mathbb{Z}$ and $b \in \mathbb{Z}\}$ where $\omega=e^{i \pi / 3}$. The origin is said to be of generation 0 , its six neighbors are of generation 1 , and so on. The set of points in $\operatorname{HL}(h, \infty)$ of generation $\leq n$ is denoted $\mathrm{HL}(h, n)$; we shall write $\operatorname{HL}(n)$ when the mesh size $h$ need not be specified. We consider $\mathrm{HL}(1 / n, n)$ as a discrete approximation grid for the unit hexagon $H$.

In an obvious way, the lattice $\mathrm{HL}(n)$ determines a triangulation of a hexagon by equilateral triangles with the lattice points as the vertices. A circle packing immersion with the combinatorics of this triangulation will be called a hexagonal circle packing immersion of $n$ generations and will be denoted $\mathrm{HCP}_{n}^{\prime \prime}$ (Figure 2.1). (Note that the Ring Lemma of [15] does not apply to circle packing immersions since the circles in a flower might overlap.) A circle packing imbedding with these combinatorics is denoted $\mathbf{H C P}_{n}^{\prime}$ (Figure 2.2). An $\mathbf{H C P}_{n}^{\prime}$ packing in which all circles have the same radius $h$ is called the regular hexagonal circle packing of $n$ generations and is denoted $\mathrm{HCP}(h, n)$ (or $\mathrm{HCP}_{n}$ if the common radius need not be specified). For example, if a circle of radius $1 / 2 n$ is centered at each point of $\operatorname{HL}(1 / n, n)$ one obtains an $\operatorname{HCP}(1 / 2 n, n)$ configuration.

It is convenient to let $\operatorname{rad} P$ denote the radius function $R$; that is, $\operatorname{rad} P(v)$ is the radius of the circle $P(v)$. The radius function for an $\mathrm{HCP}_{n}^{\prime}$ configuration is a discrete subharmonic function on the lattice $\operatorname{HL}(n)$ in the sense that its value at a point of $\mathrm{HL}(n-1)$ cannot exceed the average of its values at the six neighboring points (Barany, Furedi, and Pack [4]; [16] contains a proof of this fact and of others that will be needed from discrete potential theory). Furthermore, the reciprocal radius function $1 / \operatorname{rad} P: \operatorname{HL}(n) \rightarrow \mathbb{R}$ is also a discrete subharmonic function (see [4]). For the sake of a unified treatment we derive the maximum and minimum principles for $\operatorname{rad} P$ as consequences of the subharmonicity of $\operatorname{rad} P$ and $1 / \operatorname{rad} P$; in fact, these two principles are obvious without reference to the more quantitive property of subharmonicity.

Example (Peter Doyle). Let $c \in \mathbb{C}$. Consider the radius function $R$ on $\operatorname{HL}(1, \infty)$ defined by $R(\alpha)=\left|e^{c \alpha}\right|(\alpha=m+n \omega$ with $m, n \in \mathbb{Z}$ and $\omega=$ 


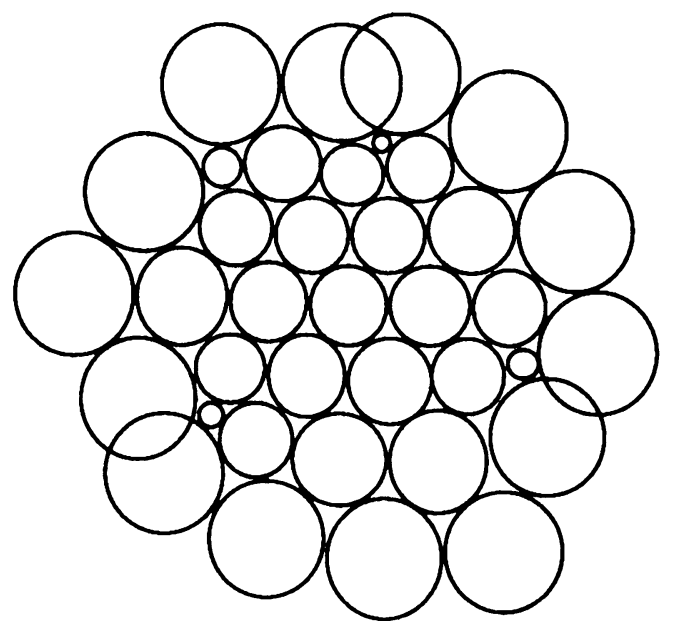

FIGURE 2.1. An $\mathrm{HCP}_{3}^{\prime \prime}$ circle packing immersion

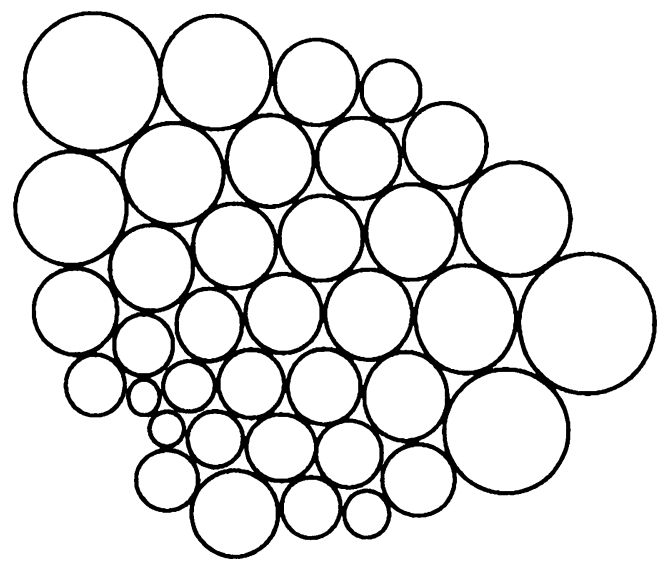

Figure 2.2. An $\mathrm{HCP}_{3}^{\prime}$ circle packing imbedding

$\left.e^{i \pi / 3}\right)$. Peter Doyle [8] observed that this radius function is flat. To see this, consider a lattice point $\alpha$ and its six neighbors $\zeta_{j}=\alpha+\omega^{j} \quad(j=0,1,2,3,4,5)$. The radii assigned to these six points by $R$ can be written

$$
\begin{aligned}
R\left(\zeta_{0}\right)=\left|e^{c}\right| R(\alpha) \equiv a R(\alpha), & & R\left(\zeta_{1}\right)=\left|e^{c \omega}\right| R(\alpha) \equiv \mathbf{R}(\alpha), \\
R\left(\zeta_{2}\right)=\left|e^{c \omega^{2}}\right| R(\alpha)=\frac{b}{a} R(\alpha), & & R\left(\zeta_{3}\right)=\left|e^{c \omega^{3}}\right| R(\alpha)=\frac{1}{a} R(\alpha), \\
R\left(\zeta_{4}\right)=\left|e^{c \omega^{4}}\right| R(\alpha)=\frac{1}{b} R(\alpha), & & R\left(\zeta_{5}\right)=\left|e^{c \omega^{5}}\right| R(\alpha)=\frac{a}{b} R(\alpha) .
\end{aligned}
$$

In the associated cone manifold there are six triangular faces determined by the seven radii $R(\alpha)$ and $R\left(\zeta_{j}\right)$. The three faces $\left(\alpha, \zeta_{0}, \zeta_{1}\right),\left(\zeta_{3}, \alpha, \zeta_{2}\right)$, and $\left(\zeta_{4}, \zeta_{5}, \alpha\right)$ are similar and hence the sum of the angles at $\alpha$ in these three faces is $\pi$. In the same way, the sum of the angles at $\alpha$ in the faces $\left(\alpha, \zeta_{1}, \zeta_{2}\right)$, $\left(\zeta_{4}, \alpha, \zeta_{3}\right)$, and $\left(\zeta_{5}, \zeta_{0}, \alpha\right)$ is seen to be $\pi$. Thus the curvature at $\alpha$ is zero and $R$ is flat. Note that in the associated circle packing immersion, the radius of any circle is the geometric mean of the radii of the surrounding six circles; that is, $\log R$ is a discrete harmonic function on $\operatorname{HL}(1, \infty)$. Figure 2.3 shows this Doyle circle packing immersion for $a=1.1, b=1.4$. 


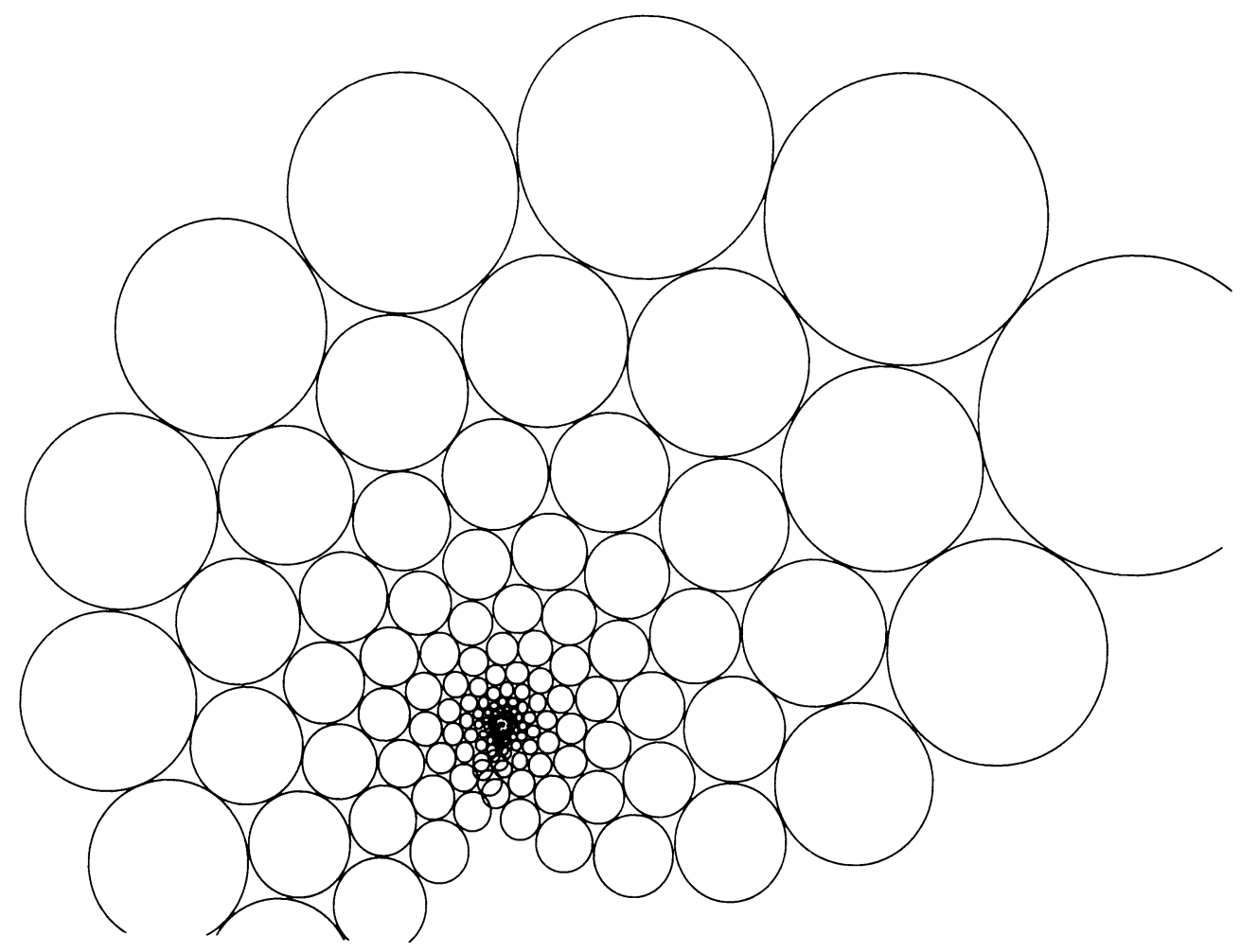

FIGURE 2.3. Doyle's packing immersion for $a=1.1$, $b=1.4$.

Next we state two results that will be needed later. Proposition 2 is credited to Andreev [2] by Thurston [19, 20] who gave it the circle packing formulation, indicated a less complicated proof (which is carried out in [14]), and gave an algorithm (Remark 2 ) for effectively computing the circle packings.

Proposition 2 (Andreev [2], Thurston [19, 20]). Let $T$ be a triangulation of the 2-sphere. Let $\tau$ be a face of $T$. Then there is a radius function $R$ defined on the vertices of $T$ which is flat at every vertex except those which belong to $\tau . R$ is uniquely determined by specifying its values at the vertices of $\tau$.

Proposition 3 (Thurston [19]). Let $T$ be a triangulation of a torus. Then there is a flat radius function $R$ defined on the vertices of $T . R$ is uniquely determined up to a scalar multiple.

In $\S 3$ we prove the following generalization of Propositions 2 and 3.

Theorem 1 (The flat radius function with boundary radii $\beta$ ). Let $T$ be a triangulation of a compact bordered surface of genus 0 or 1 with nonempty border. Let $\beta$ be a positive function defined on the border vertices of $T$. Then there exists a unique flat radius function $R$ such that $R(v)=\beta(v)$ for each border vertex $v$.

Let $P$ be an $\mathrm{HCP}_{n}^{\prime \prime}$ configuration, that is, a circle packing immersion with the combinatorics of the $\mathrm{HL}(n)$ triangulation. For $0 \leq k \leq n$, let $\max _{k} \operatorname{rad} P$ denote the maximum of $\operatorname{rad} P(v)$ over all vertices $v$ of generation $k$ in $\operatorname{HL}(n)$; 
define $\min _{k} \operatorname{rad} P$ similarly. For $q \geq 1$ define $\sigma_{n}(q)$ to be the smallest real number such that

$$
\frac{\max _{1} \operatorname{rad} P}{\min _{1} \operatorname{rad} P}-1 \leq \sigma_{n}(q)
$$

for all $\mathrm{HCP}_{n}^{\prime \prime}$ circle packing immersions $P$ which satisfy

$$
\frac{\max _{n} \operatorname{rad} P}{\min _{n} \operatorname{rad} P} \leq q
$$

Remark 1. Note that the sequence $s_{n}$ introduced in [15] can be defined in a parallel manner. Namely, $s_{n}$ is the smallest real number such that

$$
\frac{\max _{1} \operatorname{rad} P}{\min _{1} \operatorname{rad} P}-1 \leq s_{n}
$$

for all $\mathrm{HCP}_{n}^{\prime}$ circle packing imbeddings $P$.

Theorem 2. For fixed $q \geq 1, \sigma_{n}(q) \rightarrow 0$ as $n \rightarrow \infty$.

The proof of Theorem 2 appears in $\S 4$.

Let $\Omega$ be a bounded, simply-connected domain containing fixed points $z_{0}$ and $z_{1}$ (in many applications $\Omega$ will be the unit disk). Given a positive continuous function $\rho$ on $\partial \Omega$, we construct a sequence of circle packings and their radius functions as follows. For $\varepsilon>0$ sufficiently small consider $\operatorname{HCP}(\varepsilon, \infty)$, the infinite regular hexagonal circle packing of the plane by circles of radius $\varepsilon$. Starting with a circle $c_{0}$ closest to $z_{0}$, form all chains of circles from $\operatorname{HCP}(\varepsilon, \infty)$ such that the six neighbors of each circle in the chain lie in $\Omega$. Let $C_{\varepsilon}$ consist of all such circles appearing in these chains, together with all circles which are neighbors of at least one of these circles. Let $T_{\varepsilon}$ be the triangulated plane region obtained by joining the centers of all triples of tangent circles in $C_{\varepsilon}$.

Associate to $\rho$ the function $\beta$ defined on the border vertices of $T_{\varepsilon}$ as follows. If $v$ is a border vertex of $T_{\varepsilon}$ then define $\beta(v)=\varepsilon \rho\left(\zeta_{v}\right)$, where $\zeta_{v}$ is a point on $\partial \Omega$ which is closest to $v$; we frequently write $\beta \approx \varepsilon \rho$ to help recall this definition of $\beta$. Apply Theorem 1 for these boundary values $\beta$; it yields a flat radius function which, since $\Omega$ is simply connected, determines a circle packing immersion $P_{\varepsilon}$. Normalize $P_{\varepsilon}$ by requiring that $P_{\varepsilon}\left(v_{0}\right)$ is centered at the origin and $P_{\varepsilon}\left(v_{1}\right)$ is centered on the positive real axis; here $v_{0}$ is the vertex of $T_{\varepsilon}$ closest to $z_{0}$ and $v_{1}$ is the vertex of $T_{\varepsilon}$ closest to $z_{1}$. Let $r_{\varepsilon}$ be the associated radius function; that is, $r_{\varepsilon}(v)$ is the radius of the circle corresponding to $v$ in the circle packing $P_{\varepsilon}$.

We intend to discuss the convergence as $\varepsilon \rightarrow 0$ of the discrete functions $P_{\varepsilon}$ and $r_{\varepsilon}$. For that purpose it is convenient to extend their domains from the vertices of $T_{\varepsilon}$ to all of $\Omega_{\varepsilon}$, the carrier of $T_{\varepsilon}$. A piecewise linear extension will be used. The circle packing immersion $P_{\varepsilon}$ gives rise to a piecewise linear immersion $\widetilde{P}_{\varepsilon}: \Omega_{\varepsilon} \rightarrow \mathbb{C}$ as follows. Let $\widetilde{P}_{\varepsilon}$ map a vertex $v$ of $T_{\varepsilon}$ to the center of the corresponding circle $P_{\varepsilon}(v)$, and then be extended linearly to the inside of each triangle of $T_{\varepsilon}$. Linearly extend the radius function $r_{\varepsilon}$, which is defined on the vertices of $T_{\varepsilon}$, to each triangle of $T_{\varepsilon}$ thereby obtaining a piecewise linear function $\tilde{r}_{\varepsilon}: \Omega_{\varepsilon} \rightarrow \mathbb{R}_{>0}$. The next two theorems show that the circle packing immersions converge to the conformal mapping $F$ of Proposition 1, and that the ratio of the radii of image circle to preimage circle converges to $\left|F^{\prime}\right|$. The proofs will be given in $\S \S 5$ and 6 . 
Theorem 3. Let $\Omega$ be a bounded, simply-connected region and let $\rho$ be a positive continuous function on $\partial \Omega$. As above, consider the circle packing immersion $P_{\varepsilon}$ with boundary radii $\beta \approx \varepsilon \rho$, the associated piecewise linear immersion $\widetilde{P}_{\varepsilon}$ and the piecewise linear radius function $\tilde{r}_{\varepsilon}$. Let $q=\max \rho / \min \rho$. Let $K \subset \subset \Omega$ and $N=[\operatorname{dist}(K, \partial \Omega) / 2 \varepsilon]$. The $K_{\varepsilon}^{\prime}$ denote $K$ with the edges of $T_{\varepsilon}$ removed. On $K_{\varepsilon}^{\prime}$ the complex derivatives of $\widetilde{P}_{\varepsilon}$ exist and satisfy

$$
\left|\frac{\partial \widetilde{P}_{\varepsilon}}{\partial z}\right|=\frac{\tilde{r}_{\varepsilon}}{\varepsilon}+O\left(\sigma_{N}(q)\right), \quad\left|\frac{\partial \widetilde{P}_{\varepsilon}}{\partial \bar{z}}\right|=O\left(\sqrt{\sigma_{N}(q)}\right) .
$$

Corollary. Let the $K \subset \subset \Omega . L^{\infty}$ norm on $K$ of the dilation of $\widetilde{P}_{\varepsilon}$ satisfies

$$
\left\|\frac{\partial \widetilde{P}_{\varepsilon} / \partial \bar{z}}{\partial \widetilde{P}_{\varepsilon} / \partial z}\right\|_{K} \rightarrow 0 \quad \text { as } \varepsilon \rightarrow 0
$$

Theorem 4. Let $\Omega$ be a bounded, simply-connected region containing fixed points $z_{0}$ and $z_{1}$. Let $\rho$ be a positive continuous function on $\partial \Omega$. Let $F$ be the normalized conformal immersion determined by $\rho$ according to Proposition 1. As above, let $P_{\varepsilon}$ be the normalized circle packing immersion with boundary radii $\beta \approx \varepsilon \rho$ and let $r_{\varepsilon}$ be its radius function. Let $\widetilde{P}_{\varepsilon}$ and $\tilde{r}_{\varepsilon}$ be the associated piecewise linear functions. Then $\widetilde{P}_{\varepsilon}$ converges to $F$ uniformly on compacta of $\Omega$. Furthermore, $\tilde{r}_{\varepsilon} / \varepsilon$ converges to $\left|F^{\prime}\right|$ uniformly on compacta of $\Omega$.

\section{Proof of TheOREM 1}

Theorem 1 (The flat radius function with boundary radii $\beta$ ). Let $T$ be a triangulation of a compact bordered surface of genus 0 or 1 with nonempty border. Let $\beta$ be a positive function defined on the border vertices of $T$. Then there exists a unique flat radius function $R$ such that $R(v)=\beta(v)$ for each border vertex $v$.

Remark 2. For genus greater than 1 there are examples of triangulated compact surfaces with nonempty border which cannot support a flat radius function. This may be contrasted with the fact that if one is given a compact bordered Riemann surface and a metric on the border, there is a conformally equivalent flat metric on the surface which agrees with the given one on the border.

Proof. The proof of Theorem 1 will be accomplished by constructing barrier radius functions of positive and negative curvature. Label the vertices of $T$ by $v_{1}, v_{2}, \ldots, v_{V}$. Guess an initial radius function $G$ on the vertices of $T$ such that $G$ coincides with $\beta$ on the border vertices of $T$.

Complete $T$ to a triangulation $T^{*}$ of the Riemann sphere or a torus. This can be done by welding a disk to each boundary cycle and adding a vertex to each such disk. By Propositions 2 and 3, we can find a radius function defined on the vertices of $T^{*}$ which is flat at all the vertices of $T$ which do not lie on the border of $R$. Let $A$ denote the restriction of this radius function to the vertices of $T$; then we have $\kappa_{A}\left(v_{i}\right)=0$ at all interior vertices $v_{i} \in T$.

Choose a positive $\varepsilon<1$ such that $\varepsilon A<G$; that is, $\varepsilon A(v)<G(v)$ for all vertices $v \in T$. Choose $\delta>1$ such that $\delta A>G$. Construct radius functions 
$P$ and $N$ as follows.

$$
\begin{aligned}
& P\left(v_{i}\right)=\delta A\left(v_{i}\right) \text { at the interior vertices } v_{i} \in T, \\
& N\left(v_{i}\right)=\varepsilon A\left(v_{i}\right) \text { at the interior vertices } v_{i} \in T, \\
& P\left(v_{j}\right)=N\left(v_{j}\right)=\beta\left(v_{j}\right) \text { at the border vertices } v_{j} \in T .
\end{aligned}
$$

Thus $P$ and $N$ are rescaled flat radius functions on the interior vertices of $T$, while on the border vertices of $T$ both $P$ and $N$ have the values prescribed by $\beta$. It follows from the construction that $P \geq G \geq N$.

We have $\kappa_{P}\left(v_{i}\right) \geq 0$ and $\kappa_{N}\left(v_{i}\right) \leq 0$ at each interior vertex $v_{i} \in T$. Indeed, both $P$ and $N$ have zero curvature at all interior vertices of $T$ which do not have a border vertex for a neighbor. At the other interior vertices of $T$ note that if the radii assigned to the border vertices is increased, for instance, then the angles at these interior vertices in the Euclidean triangles of circle centers can only increase, and so the curvature at these vertices can only decrease.

We define the relaxation operator $\mathscr{R}_{v}$ as follows. $\mathscr{R}_{v}$ will operate on a radius function $G$ by modifying its value at the vertex $v$.

$$
\mathscr{R}_{v_{i}}: G \rightarrow G^{\prime}, \quad G^{\prime}\left(v_{j}\right)= \begin{cases}r & \text { if } j=i, \\ G\left(v_{j}\right) & \text { if } j \neq i,\end{cases}
$$

where $r$ is the unique radius which forces $\kappa_{G^{\prime}}\left(v_{i}\right)=0$. The proof of Theorem 1 is completed by the following four lemmas.

Remark 3. We shall construct the flat radius function defined on the vertices of $T$ which has the prescribed values $\beta$ on the border vertices by relaxing the initial radius function $G$ at each interior vertex of $T$ infinitely often. We refer to this algorithm as Thurston's Algorithm [20]. By Remark 2, the convergence or divergence of this algorithm depends on the combinatorial topology of $T$ if the genus is greater than 1.

Lemma 3.1. Consider the radius functions $P, G$, and $N$ defined as above. Thus $P \geq G \geq N$ at each vertex of $T$, and at each interior vertex $v_{i} \in T$ we have $\kappa_{P}\left(v_{i}\right) \geq 0$ and $\kappa_{N}\left(v_{i}\right) \leq 0$. Then for each interior vertex $v_{i} \in T$ the following inequalities hold:

$$
P \geq \mathscr{R}_{v_{i}}(P) \geq \mathscr{R}_{v_{i}}(G) \geq \mathscr{R}_{v_{i}}(N) \geq N
$$

Proof. The first inequality from the left is true because if one relaxes the radius at a vertex of positive curvature then the radius at that vertex must decrease.

To verify the second inequality note that $P \geq G$. Suppose $\mathscr{R}_{v_{i}}$ changes the radius functions $P, G$ to $P^{\prime}, G^{\prime}$ by changing the two radii $r_{i}, g_{i}$ at $v_{i}$ to $r_{i}^{\prime}, g_{i}^{\prime}$. We want to show $r_{i}^{\prime} \geq g_{i}^{\prime}$. Suppose $r_{i}^{\prime}<g_{i}^{\prime}$. Then the curvatures at $v_{i}$ satisfy $\kappa_{P^{\prime}}\left(v_{i}\right)<\kappa_{G^{\prime}}\left(v_{i}\right)$. This contradicts the fact that both of the curvatures of $P$ and $G$ at $v_{i}$ become zero after relaxation at $v_{i}$.

Lemma 3.2. At each interior vertex $v_{i} \in T, \mathscr{R}_{v_{i}}(P)$ has nonnegative curvature and $\mathscr{R}_{v_{i}}(N)$ has nonpositive curvature.

Proof. Relaxation of the nonnegative curvature radius function $P$ at the interior vertex $v_{i}$ must decrease the radius $r_{i}$ of $P$ at $v_{i}$. Thus, all of the angles at the neighboring vertices of $v_{i}$ can only decrease, whence the curvatures of $P$ at the neighboring interior vertices of $v_{i}$ can only increase. 
By repeated application of Lemmas 3.1 and 3.2 we obtain the following sequence of inequalities:

$$
\begin{gathered}
P \geq G \geq N, \\
P \geq \mathscr{R}_{v_{i}}(P) \geq \mathscr{R}_{v_{i}}(G) \geq \mathscr{R}_{v_{i}}(N) \geq N, \\
\mathscr{R}_{v_{i}}(P) \geq \mathscr{R}_{v_{j}} \circ \mathscr{R}_{v_{i}}(P) \geq \mathscr{R}_{v_{j}} \circ \mathscr{R}_{v_{i}}(G) \geq \mathscr{R}_{v_{j}} \circ \mathscr{R}_{v_{i}}(N) \geq \mathscr{R}_{v_{i}}(N),
\end{gathered}
$$

where $v_{i}, v_{j}, \ldots$ are interior vertices of $T$.

Let us write $\mathscr{R}^{1}(P)$ for $\mathscr{R}_{v_{i}}(P), \mathscr{R}^{2}(P)$ for $\mathscr{R}_{v_{j}} \circ \mathscr{R}_{v_{i}}(P)$, and so on. The radius functions $\mathscr{R}^{j}(P), j=1,2, \ldots$, are monotone decreasing and bounded below by $N$. Let $P_{0}=\inf _{j}\left\{\mathscr{R}^{j}(P)\right\}$. The radius functions $\mathscr{R}^{j}(N)$, $j=1,2, \ldots$, are monotone increasing and bounded above by $P$. Let $N_{0}=$ $\sup _{j}\left\{\mathscr{R}^{j}(N)\right\}$. Then $P_{0} \geq \mathscr{R}^{\infty}(G) \geq N_{0}$, where $\mathscr{R}^{\infty}(G)$ is any subsequential limit of the iterates $\mathscr{R}^{j}(G), j=1,2, \ldots$.

Lemma 3.3. Consider the radius functions $P$ and $N$ defined above. Write $\mathscr{R}^{i}(P)$ for $\mathscr{R}_{v_{i}}(P), \mathscr{R}^{2}(P)$ for $\mathscr{R}_{v_{j}} \circ \mathscr{R}_{v_{i}}(P)$, and so on. Let $P_{0}=\inf _{j}\left\{\mathscr{R}^{j}(P)\right\}$ and $N_{0}=\sup _{j}\left\{\mathscr{R}^{j}(N)\right\}$. Then both $P_{0}$ and $N_{0}$ have zero curvature at each interior vertex of $T$.

Proof. Given a vertex $v$ and radii $r_{1}, r_{2}, \ldots, r_{m}$ at each vertex in the star of $v$, there is unique radius $r$ at $v$ which will make this configuration have curvature 0 at $v$; furthermore $r=r\left(r_{1}, r_{2}, \ldots, r_{m}\right)$ is a continuous function.

Now let $r$ denote the radius at the vertex $v$ in the radius function $P_{0}$. Let $r_{1}, r_{2}, \ldots, r_{m}$ denote the radii in $P_{0}$ at the vertices in the star of $v$. Let $r^{j}$ denote the radius at the vertex $v$ in the radius function $\mathscr{R}^{j}(P)$. Let $r^{j_{1}}, r^{j_{2}}, \ldots, r^{j_{m}}$ denote the radii in $\mathscr{R}^{j}(P)$ at the vertices in the star of $v$.

If, for example, $P_{0}$ has strictly positive curvature at $v$ then $\mathscr{R}_{v}\left(P_{0}\right)$ changes the radius $r$ at $v$ to $r^{\prime}$, where $r^{\prime}=r-\varepsilon$ for some $\varepsilon>0$. Choose $\delta>0$ such that if $\left\|\left(\rho_{1}, \rho_{2}, \ldots, \rho_{m}\right)-\left(\rho_{1}^{\prime}, \rho_{2}^{\prime}, \ldots, \rho_{m}^{\prime}\right)\right\|<\delta$ then $\mid r\left(\rho_{1}, \rho_{2}, \ldots, \rho_{m}\right)-$ $r\left(\rho_{1}^{\prime}, \rho_{2}^{\prime}, \ldots, \rho_{m}^{\prime}\right) \mid<\varepsilon / 2$. Choose $M>0$ such that for all $j>M$,

$$
\left\|\left(r^{j_{1}}, r^{j_{2}}, \ldots, r^{j_{m}}\right)-\left(r_{1}, r_{2}, \ldots, r_{m}\right)\right\|<\delta .
$$

Then there exists a positive integer $d$ such that $\left|r^{j+d}-r^{\prime}\right|<\varepsilon / 2$, where $d$ is chosen such that $\mathscr{R}^{M+d}(P)$ relaxes at $v$. Thus $\left\{r^{j_{i}}\right\}$ converges to some number less than $r^{\prime}+\varepsilon / 2<r$. This contradicts the fact that $r=\inf _{j}\left\{r^{j}\right\}$.

At this point we know that

$$
\inf _{j}\left\{\mathscr{R}^{j}(P)\right\}=P_{0} \geq \mathscr{R}^{\infty}(G) \geq N_{0}=\sup _{j}\left\{\mathscr{R}^{j}(N)\right\}
$$

where $R^{\infty}(G)$ is any subsequential limit of the iterates $\mathscr{R}^{j}(G), j=1,2, \ldots$, of the initial radius function $G$, both $P_{0}$ and $N_{0}$ have zero curvature at the interior vertices of $T$, and $P_{0}$ coincides with $N_{0}$ on the border vertices of $T$.

Lemma 3.4. $P_{0}=N_{0}$.

Proof. This proof was suggested to us by Carl FitzGerald. Suppose $P_{0} \neq N_{0}$; that is, $P_{0}\left(v_{j}\right) \neq N_{0}\left(v_{j}\right)$ at some interior vertex $v_{j} \in T$. Let $P_{0}\left(v_{\alpha}\right) / N_{0}\left(v_{\alpha}\right)>1$ maximize $P_{0}\left(v_{i}\right) / N_{0}\left(v_{i}\right)$ over all vertices $v_{i} \in T$. Then at each neighboring vertex $v_{j}$ of $v_{\alpha}$ we must have $P_{0}\left(v_{j}\right) / N_{0}\left(v_{j}\right)=P_{0}\left(v_{\alpha}\right) / N_{0}\left(v_{\alpha}\right)$, for otherwise 
$P_{0}\left(v_{j}\right) / N_{0}\left(v_{j}\right)<P_{0}\left(v_{\alpha}\right) / N_{0}\left(v_{\alpha}\right)$ and then either $P_{0}$ or $N_{0}$ would not have zero curvature at the interior vertex $v_{\alpha}$. Repeating this argument on a chain of vertices to the border of $T$ yields $P_{0}\left(v_{k}\right)>N_{0}\left(v_{k}\right)$ at some border vertex $v_{k} \in T$. This contradicts the fact that $P_{0}$ coincides with $N_{0}$ at the border vertices of $T$.

\section{Proof of Theorem 2}

The constants $\sigma_{n}(q)$ were defined in (2.1) and (2.2).

Theorem 2. For fixed $q \geq 1, \sigma_{n}(q) \rightarrow 0$ as $n \rightarrow \infty$.

Proof. Let $P_{n}$ be an $\mathrm{HCP}_{n}^{\prime \prime}$ configuration, that is, a circle packing immersion with the combinatorics of the $\mathrm{HL}(n)$ triangulation. Assume that $(2.2)$ holds, that is, the ratio of maximum to minimum radii of the circles of generation $n$ is bounded above by $q$. We may assume that the generation 0 circle has radius 1. As mentioned in $\S 2$, the associated radius function $r_{n}: \operatorname{HL}(1, n) \rightarrow \mathbb{R}_{>0}$ and its reciprocal $1 / r_{n}: \operatorname{HL}(1, n) \rightarrow \mathbb{R}_{>0}$ are discrete subharmonic functions.

By Theorem 3.2 of [16] there is a fundamental potential $\lambda: \operatorname{HL}(1, \infty) \rightarrow$ $\mathbb{R}$ which is harmonic at all lattice points except the origin and which can be normalized to satisfy $\lambda(\alpha)-\log |\alpha| \rightarrow 0$ as $\alpha \rightarrow \infty$. For $n$ sufficiently large and $\alpha \in \operatorname{HL}(1, n)$ we have

$$
r_{n}(\alpha) \leq \frac{q-1}{\min _{n} \lambda-\lambda(0)}(\lambda(\alpha)-\lambda(0))+1 ;
$$

indeed, the right side is harmonic on $\operatorname{HL}(1, n)-\{0\}$, is equal to 1 at $\alpha=0$, and its minimum over all $\alpha$ of generation $n$ is $q$.

The function $1 / r_{n}$ is a discrete subharmonic function on $\operatorname{HL}(1, n), 1 / r_{n}(0)$ $=1$, and $1 / r_{n}$ is bounded above by $q$. Hence

$$
\frac{1}{r_{n}(\alpha)} \leq \frac{q-1}{\min _{n} \lambda-\lambda(0)}(\lambda(\alpha)-\lambda(0))+1 .
$$

From (4.1), (4.2) we obtain, for $\alpha$ fixed and $n \rightarrow \infty$,

$$
1+o(1) \leq r_{n}(\alpha) \leq 1+o(1)
$$

where the terms $o(1)$ depend only on $n, \alpha$, and $q$, and not on the particular radius function $r_{n}$. From (4.3) we see that

$$
\frac{\max _{1} r_{n}}{\min _{1} r_{n}} \leq 1+o(1)
$$

which proves that $\sigma_{n}(q) \leq(q-1) O(1 / \log n)$.

\section{Proof of Theorem 3}

Theorem 3. Let $\Omega$ be a bounded, simply-connected region and let $\rho$ be a positive continuous function on $\partial \Omega$. As above, consider the circle packing immersion $P_{\varepsilon}$ with boundary radii $\beta \approx \varepsilon \rho$, the associated piecewise linear immersion $\widetilde{P}_{\varepsilon}$ and the piecewise linear radius function $\tilde{r}_{\varepsilon}$. Let $q=\max \rho / \min \rho$. Let $K \subset \subset \Omega$ and $N=[\operatorname{dist}(K, \partial \Omega) / 2 \varepsilon]$. Let $K_{\varepsilon}^{\prime}$ denote $K$ with the edges of $T_{\varepsilon}$ removed. On $K_{\varepsilon}^{\prime}$ the complex derivatives of $\widetilde{P}_{\varepsilon}$ exist and satisfy

$$
\left|\frac{\partial \widetilde{P}_{\varepsilon}}{\partial z}\right|=\frac{\tilde{r}_{\varepsilon}}{\varepsilon}+O\left(\sigma_{N}(q)\right), \quad\left|\frac{\partial \widetilde{P}_{\varepsilon}}{\partial \bar{z}}\right|=O\left(\sqrt{\sigma_{N}(q)}\right) .
$$


Proof. By Lemma 6.4 of [16] we find

$$
\begin{aligned}
2 \varepsilon^{2}\left|\frac{\partial \widetilde{P}_{\varepsilon}}{\partial z}\right|^{2}= & \frac{1}{12}\left[\left(R_{1}+R_{2}\right)^{2}+\left(R_{2}+R_{3}\right)^{2}+\left(R_{3}+R_{1}\right)^{2}\right] \\
& +\frac{1}{\sqrt{3}}\left[R_{1} R_{2} R_{3}\left(R_{1}+R_{2}+R_{3}\right)\right]^{1 / 2} \\
2 \varepsilon^{2}\left|\frac{\partial \widetilde{P}_{\varepsilon}}{\partial \bar{z}}\right|^{2}= & \frac{1}{12}\left[\left(R_{1}+R_{2}\right)^{2}+\left(R_{2}+R_{3}\right)^{2}+\left(R_{3}+R_{1}\right)^{2}\right] \\
& -\frac{1}{\sqrt{3}}\left[R_{1} R_{2} R_{3}\left(R_{1}+R_{2}+R_{3}\right)\right]^{1 / 2}
\end{aligned}
$$

where $R_{1}, R_{2}, R_{3}$ are the radii of three mutually tangent circles in the circle packing $P_{\varepsilon}$ and where the left hand sides are evaluated at a point inside the equilateral triangle formed by the preimages of the three circles. If this point lies in the fixed compact subset $K$ of $\Omega$, then each of $R_{1}, R_{2}, R_{3}$ is the generation zero circle of a circle packing immersion of $N=[\operatorname{dist}(K, \partial \Omega) / 2 \varepsilon]$ generations. On the $N$ th generation of this subpacking the maximum and minimum radii have a ratio which cannot exceed $q$, the ratio on the boundary. By the definition of $\sigma_{n}(q)$ we have $R_{2}=R_{1}\left(1+O\left(\sigma_{N}(q)\right)\right), R_{3}=R_{1}\left(1+O\left(\sigma_{N}(q)\right)\right)$, where $O\left(\sigma_{N}(q)\right) / \sigma_{N}(q)$ is bounded (here, in fact, by 1 ) as $N \rightarrow \infty$. to

By Theorem 2, $\sigma_{n}(q) \rightarrow 0$ as $n \rightarrow \infty$. Therefore, equations (5.1) simplify

$$
2 \varepsilon^{2}\left|\frac{\partial \widetilde{P}_{\varepsilon}}{\partial z}\right|^{2}=R_{1}^{2}\left(2+O\left(\sigma_{N}(q)\right)\right), \quad 2 \varepsilon^{2}\left|\frac{\partial \widetilde{P}_{\varepsilon}}{\partial \bar{z}}\right|^{2}=R_{1}^{2} O\left(\sigma_{N}(q)\right) .
$$

Now $R_{1}$ is bounded above by the maximum of the radii in the circle packing $P_{\varepsilon}$, and this maximum occurs on $\partial T_{\varepsilon}$. Thus $R_{1} \leq \varepsilon \max \rho$; we write $R_{1}=O(\varepsilon)$ since $\rho$ is fixed. Also, the piecewise linear extension satisfies $\tilde{r}_{\varepsilon}(z)=R_{1}(1+$ $\left.O\left(\sigma_{N}(q)\right)\right)$ for $z$ inside the preimage triangle of centers. We therefore have

$$
\left|\frac{\partial \widetilde{P}_{\varepsilon}}{\partial z}\right|^{2}=\left(\frac{\tilde{r}_{\varepsilon}}{\varepsilon}\right)^{2}+O\left(\sigma_{N}(q)\right), \quad\left|\frac{\partial \widetilde{P}_{\varepsilon}}{\partial \bar{z}}\right|^{2}=O\left(\sigma_{N}(q)\right)
$$

and the theorem follows.

Corollary. Let $K \subset \subset \Omega$. On $K$, the $L^{\infty}$ norm of the dilation of $\widetilde{P}_{\varepsilon}$ satisfies

$$
\left\|\frac{\partial \widetilde{P}_{\varepsilon} / \partial \bar{z}}{\partial \widetilde{P}_{\varepsilon} / \partial z}\right\|_{K} \rightarrow 0 \quad \text { as } \varepsilon \rightarrow 0
$$

Proof. Since $1 / \tilde{r}_{\varepsilon}$ is discrete subharmonic on the lattice of vertices, it satisfies $\tilde{r}_{\varepsilon} \geq \min \beta$. Hence $\tilde{r}_{\varepsilon} / \varepsilon \geq \min \rho$. Therefore, by Theorem 3 , the denominator in (5.4) is bounded away from zero and the numerator converges to zero.

\section{Proof of Theorem 4}

Theorem 4. Let $\Omega$ be a bounded, simply-connected region containing fixed points $z_{0}$ and $z_{1}$. Let $\rho$ be a positive continuous function on $\partial \Omega$. Let $F$ be the normalized conformal immersion determined by $\rho$ according to Proposition 1. 
As above, let $P_{\varepsilon}$ be the normalized circle packing immersion with boundary radii $\beta \approx \varepsilon \rho$ and let $r_{\varepsilon}$ be it radius function. Let $\widetilde{P}_{\varepsilon}$ and $\tilde{r}_{\varepsilon}$ be the associated piecewise linear functions. Then $\widetilde{P}_{\varepsilon}$ converges to $F$ uniformly on compacta of $\Omega$. Furthermore, $\tilde{r}_{\varepsilon} / \varepsilon$ converges to $\left|F^{\prime}\right|$ uniformly on compacta of $\Omega$.

Proof. Recall the construction of $P_{\varepsilon}$ from $\rho$. For $\varepsilon>0$ sufficiently small we consider the regular hexagonal circle packing $\operatorname{HCP}(\varepsilon, \infty)$ of the plane by circles of radius $\varepsilon$. Starting at the circle $c_{0}$ closest to $z_{0}$, form all chains of circles from $\operatorname{HCP}(\varepsilon, \infty)$ such that the six neighbors of each circle in the chain lie in $\Omega$. Let $C_{\varepsilon}$ consist of all such circles appearing in these chains, together with all circles which are neighbors of at least one of these circles. Let $T_{\varepsilon}$ be the nerve of $C_{\varepsilon}$.

Associate to $\rho$ the function $\beta \approx \varepsilon \rho$ defined on the border vertices of $T_{\varepsilon}$ as follows. If $v$ is a border vertex of $T_{\varepsilon}$ then define $\beta(v)=\varepsilon \rho\left(\zeta_{v}\right)$, where $\zeta_{v}$ is a point on $\partial \Omega$ which is closest to $v$. Apply Theorem 1 for these boundary values to obtain a circle packing immersion $P_{\varepsilon}$. Normalize $P_{\varepsilon}$ by requiring that $P_{\varepsilon}\left(v_{0}\right)$ is centered at the origin and $P_{\varepsilon}\left(v_{1}\right)$ is centered on the positive real axis, where $v_{0}$ is a vertex of $T_{\varepsilon}$ closest to $z_{0}$ and $v_{1}$ is a vertex of $T_{\varepsilon}$ closest to $z_{1}$. Let $r_{\varepsilon}$ be the associated radius function; that is, $r_{\varepsilon}(v)$ is the radius of the circle corresponding to $v$ in the circle packing immersion $P_{\varepsilon}, r_{\varepsilon}$ coincides with $\beta$ on the border vertices of $T_{\varepsilon}$.

The circle packing immersion $P_{\varepsilon}$ determines a discrete mapping of the vertices of $T_{\varepsilon}$ into $\mathbb{C}$ by mapping a vertex of $T_{\varepsilon}$ to the center of the corresponding circle. The piecewise linear extension of this mapping is $\widetilde{P}_{\varepsilon}: \Omega_{\varepsilon} \rightarrow \mathbb{C}$, where $\Omega_{\varepsilon}$ denotes the carrier of $T_{\varepsilon}$. The normalization implies that $\widetilde{P}_{\varepsilon}\left(z_{0}\right) \rightarrow 0$ and $\widetilde{P}_{\varepsilon}\left(z_{1}\right)$ tends to the nonnegative real axis as $\varepsilon \rightarrow 0$.

Let $q=\max \rho / \min \rho$. The complex derivatives of $\widetilde{P}_{\varepsilon}$ satisfy Theorem 3 . Hence, since $\sigma_{n}(q) \rightarrow 0$ as $n \rightarrow \infty$,

$$
\left|\frac{\partial \widetilde{P}_{\varepsilon}}{\partial z}\right|-\frac{\tilde{r}_{\varepsilon}}{\varepsilon} \rightarrow 0, \quad\left|\frac{\partial \widetilde{P}_{\varepsilon}}{\partial \bar{z}}\right| \rightarrow 0
$$

in the $L_{\infty}$ norm on compact subsets of $\Omega$.

Let $d_{\max }$ denote the largest distance from $z_{0}$ to $\partial \Omega$ and let $N=d_{\max } / 2 \varepsilon$. $\left|\widetilde{P}_{\varepsilon}\right|$ attains its maximum on the border vertices of $T_{\varepsilon}$, so an upper bound for $\left\|\widetilde{P}_{\varepsilon}\right\|_{\Omega}$ is $N$ times the largest diameter of the circles of $P_{\varepsilon}$. Since each circle of $P_{\varepsilon}$ has radius at most $M \varepsilon$ where $M=\max \rho$, we obtain $\left\|\widetilde{P}_{\varepsilon}\right\|_{\Omega} \leq M d_{\max }$.

A family of $K$-quasiconformal immersions is uniformly equicontinuous. The family $\left\{\widetilde{P}_{\varepsilon}\right\}$ is quasiconformal on compacta (see the corollary to Theorem 3 ) and is also uniformly bounded. Hence it is normal. By taking a convergent subsequence if necessary, consider $\widetilde{P}_{\varepsilon} \rightarrow G$. It follows from (6.1) that $G$ is holomorphic on the interior of $\Omega$ and $\tilde{r}_{\varepsilon} / \varepsilon \rightarrow\left|G^{\prime}\right|$ almost everywhere in $\Omega$. This can be seen, for example, by first writing $\tilde{P}_{\varepsilon}=Q_{\varepsilon} \circ \varphi_{\varepsilon}$ with $\varphi_{\varepsilon}$ a quasiconformal homeomorphism and $Q_{\varepsilon}$ a conformal immersion. By passing to another subsequence if necessary, we can assume $Q_{\varepsilon} \rightarrow Q, \varphi_{\varepsilon} \rightarrow \varphi$, and $G=Q \circ \varphi$. That $G$ has complex dilation zero almost everywhere then follows from (6.1) and [6]; see also [13, p. 187, Theorem 5.2]. From (6.1) and [13, p. 216, Theorem 5.3] it then follows that $\tilde{r}_{\varepsilon} / \varepsilon \rightarrow\left|G^{\prime}\right|$ in $L_{p}$ for some $p>2$ and hence, by passing to a subsequence if necessary, almost everywhere in $\Omega$. 
\{One can also arrive at these conclusions by using (6.1) and its implication that $\partial \widetilde{P}_{\varepsilon} / \partial z$ is uniformly bounded in $L_{\infty}$ to prove BMO convergence of the complex derivatives of $\widetilde{P}_{\varepsilon}$ to those of $G$ as in [17].\}

We have $\widetilde{P}_{\varepsilon} \rightarrow G$ uniformly on compacta of $\Omega$ and $\tilde{r}_{\varepsilon} / \varepsilon \rightarrow\left|G^{\prime}\right|$ almost everywhere in $\Omega$. The restriction of $\varepsilon / \tilde{r}_{\varepsilon}$ is a discrete subharmonic on the vertices of $T_{\varepsilon}$ and is bounded above to $1 / \min \rho$ on the boundary vertices of $T_{\varepsilon}$. By the maximum principle $\varepsilon / \tilde{r}_{\varepsilon} \leq 1 / \min \rho$ on all of the vertices of $T_{\varepsilon}$ and hence $\tilde{r}_{\varepsilon} / \varepsilon \geq \min \rho$. Since $\tilde{r}_{\varepsilon} / \varepsilon$ remains bounded away from zero on $\Omega_{\varepsilon}$, $G$ is nonconstant and conformal.

Let us show that the almost everywhere convergence of $\tilde{r}_{\varepsilon} / \varepsilon$ is actually uniform on compacta. A winding number argument shows that the uniform convergence on compacta $\widetilde{P}_{\varepsilon} \rightarrow G$ implies that each point in $\Omega$ has a neighborhood in which $G$ and $\widetilde{P}_{\varepsilon}$ are schlicht for all sufficiently small $\varepsilon$. If we restrict ourselves to this neighborhood we are dealing with a sequence of circle packing embeddings $\operatorname{HCP}^{\prime}(\varepsilon,[\delta / 2 \varepsilon])$ for some fixed $\delta>0$, which converge to a conformal embedding $G$. By the result of He [10] (combine He's result with [17, Theorems 5,8]), $\left|G^{\prime}\right|$ is the limit uniformly on compacta of $\tilde{r}_{\varepsilon} / \varepsilon$.

We now know $\widetilde{P}_{\varepsilon} \rightarrow G$ and $\tilde{r}_{\varepsilon} / \varepsilon \rightarrow\left|G^{\prime}\right|$; in both cases the convergence is uniform on compacta of $\Omega$. We wish to show that $\left|G^{\prime}\right|$ extends continuously to $\partial \Omega$ and takes the value $\rho$ there. Let $b_{\varepsilon}: \mathrm{HL}_{\varepsilon} \rightarrow \mathbb{R}$ be the discrete harmonic function on the vertices of $T_{\varepsilon}$ with boundary values obtained from $\rho$ at a nearest point on $\partial \Omega$. Let $\tilde{b}_{\varepsilon}$ be the piecewise linear extension of $b_{\varepsilon}$. Let $b: \mathrm{Cl}(\Omega) \rightarrow \mathbb{R}$ be the classical solution to the Dirichlet problem for $\Omega$ with boundary values $\rho$. Then $\tilde{b}_{\varepsilon} \rightarrow b$ uniformly on compacta of $\Omega$ (see the footnote to Theorem 4, §2). For $z \in \Omega_{\varepsilon}$ we have

$$
\tilde{r}_{\varepsilon}(z) / \varepsilon \leq \tilde{b}_{\varepsilon}(z)
$$

since the discrete restriction of the left-hand term is discrete subharmonic on the vertices of $T_{\varepsilon}$ and equal to the right side on the boundary vertices of $T_{\varepsilon}$. Let $\varepsilon \rightarrow 0$ and obtain $\left|G^{\prime}(z)\right| \leq b(z)$. For $\zeta \in \partial \Omega$ we may conclude $\lim \sup _{z \rightarrow \zeta}\left|G^{\prime}(z)\right| \leq \rho(\zeta)$.

Let $c_{\varepsilon}: T_{\varepsilon} \rightarrow \mathbb{R}$ be the discrete harmonic function with boundary values obtained from $1 / \rho$ at a nearest point on $\partial \Omega$. Let $\tilde{c}_{\varepsilon}$ be the piecewise linear extension of $c_{\varepsilon}$. Let $c: \mathrm{Cl}(\Omega) \rightarrow \mathbb{R}$ be the classical solution to the Dirichlet problem for $\Omega$ with boundary values $1 / \rho$. Then $\tilde{c}_{\varepsilon} \rightarrow c$ uniformly on compacta of $\Omega$ (see pp. 281-282 of [3]). For $z \in \Omega_{\varepsilon}$ we have

$$
\varepsilon / \tilde{r}_{\varepsilon}(z) \leq \tilde{c}_{\varepsilon}(z)
$$

because the restriction of $\varepsilon / \tilde{r}_{\varepsilon}(z)$ is discrete subharmonic on the vertices of $T_{\varepsilon}$ and equal to $\tilde{c}_{\varepsilon}(z)$ on the boundary vertices. This implies $1 /\left|G^{\prime}(z)\right| \leq c(z)$. For $\zeta \in \partial \Omega$ we may conclude

$$
\limsup _{z \rightarrow \zeta} \frac{1}{\left|G^{\prime}(z)\right|} \leq c(\zeta)=\frac{1}{\rho(\zeta)}
$$

and hence $\rho(\zeta) \leq \liminf _{z \rightarrow \zeta}\left|G^{\prime}(z)\right|$. This proves

$$
\lim _{z \rightarrow \zeta}\left|G^{\prime}(z)\right|=\rho(\zeta) .
$$


Thus the conformal immersions $F$ and $G$ have the same derivative modulus on $\partial \Omega$. The normalization that $z_{0}$ is sent to 0 and $z_{1}$ to the positive real axis implies $G \equiv F$. This completes the proof of Theorem 4 .

\section{REFERENCES}

1. Dov Aharonov, The hexagonal packing lemma and discrete potential theory, Canad. J. Math. 33 (1990), 247-252.

2. E. M. Andreev, On convex polyhedra of finite volume in Lobacevskii space, Mat. Sb. (N.S.) 83 (1970), 256-260; English transl. in Math. U.S.S.R.-Sb. 12 (1979), 255-259.

3. I. Babǔska, M. Práger, and E. Vitásek, Numerical processes in differential equations, Wiley, London, 1966, $351 \mathrm{pp}$.

4. I. Bárány, Z. Füredi, and J. Pach, Discrete convex functions and proof of the six circle conjecture of Fejes Tóth, Canad. J. Math. 36 (1984), 569-576.

5. A. Beardon and K. Stephenson, The finite Schwarz-Pick lemma, preprint.

6. L. Bers, On a theorem of Mori and the definition of quasiconformality, Trans. Amer. Math. Soc. 84 (1957), 78-84.

7. I. Carter, Circle packing and conformal mapping, Ph.D. dissertation, Univ. of California at San Diego, 1989.

8. P. Doyle, Oral communication.

9. L. J. Hanson, On the Rodin and Sullivan ring lemma, Complex Variables Theory Appl. 10 (1988), 23-30.

10. Zheng-Xu He, An estimate for hexagonal circle packings, J. Differential Geom. (to appear).

11. P. Henrici, Applied and computational complex analysis. Vol. III, Wiley, New York and London, 1986, $637 \mathrm{pp}$.

12. F. John, A criterion for univalency brought up to date, Comm. Pure Appl. Math. 29 (1976), 293-295.

13. O. Lehto and K. I. Virtanen, Quasiconformal mappings in the plane, Springer-Verlag, New York and Berlin, 1973.

14. A. Marden and B. Rodin, On Thurston's formulation and proof of Andreev's theorem, preprint.

15. B. Rodin and D. Sullivan, The convergence of circle packings to the Riemann mapping, J. Differential Geom. 26 (1987), 349-360.

16. B. Rodin, Schwarz's lemma for circle packings, Invent. Math. 89 (1987), 271-289.

17. __, Schwarz's lemma for circle packings. II, J. Differential Geom. 29 (1989).

18. J. F. Thompson (editor), Numerical grid generation, North-Holland, New York and Amsterdam, 1982.

19. W. P. Thurston, The geometry and topology of 3-manifolds, Princeton Univ. Notes, Princeton, N.J., 1980.

20. __ The finite Riemann mapping theorem, Invited Address, Internat. Sympos. in Celebration of the Proof of the Bierberbach Conjecture, Purdue University, March 1985.

21. S. E. Warschawski, On the solution of the Lichtenstein-Gershgorin integral equation in conformal mapping. I. Theory, Nat. Bur. Standards Appl. Math. Ser. 42 (1955), 7-30.

Department of Mathematics, Humboldt State University, Arcata, California 95521

Department of Mathematics, University of California, San Diego, la Jolla, CaliforNIA 92093

E-mail address: brodin@ucsd.edu 